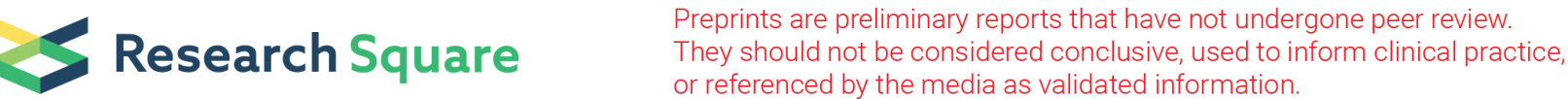

\section{Ventricle Contact Is Associated With Higher 11C Methionine PET Uptake in Glioblastoma}

\section{Bart Roelf Jan van Dijken ( $\sim$ b.r.j.van.dijken@umcg.nl )}

University Medical Centre Groningen: Universitair Medisch Centrum Groningen https://orcid.org/00000002-4804-7276

\section{Bram Schuuring}

UMCG: Universitair Medisch Centrum Groningen

\section{Hanne-Rinck Jeltema}

UMCG: Universitair Medisch Centrum Groningen

\section{Peter Jan van Laar}

Ziekenhuisgroep Twente ZGT: Ziekenhuisgroep Twente

\section{Roelien H Enting}

UMCG: Universitair Medisch Centrum Groningen

\section{Rudi A.J.O. Dierckx}

UMCG: Universitair Medisch Centrum Groningen

Gilles N. Stormezand

UMCG: Universitair Medisch Centrum Groningen

\section{Anouk van der Hoorn}

UMCG: Universitair Medisch Centrum Groningen

\section{Research Article}

Keywords: Glioblastoma, 11C Methionine PET, Ventricle contact, Subventricular zone

Posted Date: February 23rd, 2021

DOI: https://doi.org/10.21203/rs.3.rs-251550/v1

License: (c) (i) This work is licensed under a Creative Commons Attribution 4.0 International License.

Read Full License 


\section{Abstract}

Introduction: Ventricle contact is associated with a worse prognosis and more aggressive tumor characteristics in glioblastoma (GBM). This is hypothesized to be a result of neural stem cells located around the lateral ventricles, in the subventricular zone. 11C methionine Positron Emission Tomography (metPET) is an indicator for increased proliferation, as it shows uptake of methionine, an amino acid needed for protein synthesis. This study is the first to study metPET characteristics of GBM in relation to ventricle contact.

Methods: 12 patients with IDH wild type GBM a were included. Using MRI, the following regions were determined: primary tumor (defined as contrast enhancing lesion on T1) and peritumoral edema (defined as edema visible on FLAIR excluding the enhancement). PET parameters in these areas were extracted using PET fused with MRI imaging. Parameters extracted from the PET included: maximum and mean tumor-to-normal ratio (TNRmax and TNRmean) and metabolic tumor volume (MTV).

Results: TNRmean of the primary tumor showed significantly higher values for the ventricle contacting group compared to the non-contacting group (4.44 vs 2.67, $\mathrm{p}=0.015)$. Other metPET parameters suggested higher values for the ventricle contacting group, but these differences did not reach statistical significance.

Conclusion: GBM with ventricle contact show a higher methionine uptake and thus increased proliferation compared with GBM without ventricle contact. This might explain survival differences and should be considered in treatment decisions.

\section{Introduction}

Glioblastoma (GBM) is a high-grade primary brain malignancy with a dire prognosis. Despite adequate treatment median survival ranges from 12 to 18 months [1,2]. This short life expectancy, is related to the almost certain recurrence of GBM, which is due to the infiltrative nature of the tumor [3-5]. Recently, it was demonstrated that GBM with ventricle contact have a worse prognosis when compared to GBM without contact. A meta-analysis showed that ventricle contact is associated with lower survival independent of other prognostic influences [6].

The subventricular zone is an important neural stem cell containing brain region and hypothesized to play a role in the tumorigenesis of GBM $[7,8]$. Although ventricle contact has not yet been shown to be related to a specifically different cell lineage in this regard, it has been associated with more therapy resistance [9]. In addition, ventricle contacting GBMs have been shown to be more often multifocal and recur further away from the primary tumor, both features being associated with poorer survival $[6,10]$. These results seem to suggest that, although a difference in cell of origin has not yet been demonstrated, ventricle contact is associated with more aggressive tumor behavior. We hypothesized that because of their location, ventricle contacting GBM could be more prone to stem cell influence and take on more aggressive characteristics, including increased proliferation, than non-contacting GBMs. 
MR imaging, the most frequently used imaging modality in studies of GBM, provides several indirect indicators of proliferation, such as tumor size and contrast enhancement. However, neither of these parameters give a direct indication of biological activity of the tumor and are thus limited in appreciating aggressiveness and proliferation.

11C methionine PET (metPET), a widely employed amino acid tracer, has been shown to indirectly reflect tumor proliferation, as methionine uptake is associated with protein synthesis [11-13]. However, to our best knowledge, studies evaluating metPET characteristics of GBM in relation to ventricle contact have not yet been performed. We hypothesize that a more aggressive behavior of ventricle contacting GBM will be shown by a higher proliferation indicated by metPET.

\section{Methods}

\section{Patient population}

Data for this study were acquired retrospectively from patient files in the University Medical Centre of Groningen (UMCG) in the Netherlands from 2011-2019. The study has been approved by the institutional review board and the need for written informed consent was waived. We included patients with isocitrate dehydrogenase wildtype (IDHwt) GBM, following the most recent EANO (European Association of NeuroOncology) guidelines [14]. Patients were only eligible for inclusion if they conformed to the following inclusion criteria: 1) IDHwt GBM was confirmed by pathological report, 2) preoperative/prebiopsy metPET imaging was available and 3) preoperative MRI with at least T1 post-contrast and T2 FLAIR sequences was available. The MRI was required to assess the location of the tumor and to determine the peritumoral area. Exclusion criteria included 1) no contrast enhancing lesion on MRI, 2) other neurological malignant processes and 3) age below 18. Included patients were first scored on whether the tumors showed ventricle contact by BS and BVD independently, after which an inter-rater agreement score was calculated using a Cohen's Kappa score [15]. Conflicting scores were settled by AVDH, a neuro-radiologist with more than 5 years of experience in neuro oncology. Ventricle contact was determined by the extent of the contrast enhancing lesion on the T1 post-contrast sequence on MRI. This was in line with previous studies $[16,17]$. The date of diagnosis was defined as the day of the biopsy or operation that confirmed the diagnosis via pathological examination.

\section{MRI and PET acquisition}

Both MRI and PET scans were extracted from the medical files of the included patients. MRI imaging was performed on several different types of machines, from different manufacturers. All MRI were performed on 1.5T or 3.0T. The imaging protocols at least included a 3D post-contrast T1 sequence (repetition time [TR] 2000-2250 msec, echo time [TE] 2.67-3.40 msec, inversion time [TI] 850-900 msec, slice thickness $1 \mathrm{~mm}$, no slice gap, voxel size $1 \times 1 \times 1 \mathrm{~mm}$ ) and fluid-attenuated inversion recovery (FLAIR) sequence (TR 5000-11000 msec, TE 87-337 msec, TI 1800-2800 msec, slice thickness 1-5 mm, no slice gap). Most protocols also included axial T1 pre-contrast, T2, DWI. Models that were used for scanning included Philips ingenia, Siemens aera, Siemens sonata and Siemens avanto. All PET imaging was performed in 
the UMCG, using either a Siemens Biograph mCT $(\mathrm{N}=10)$ or PET-HR+ $(\mathrm{N}=3)$ scanner (Siemens, Knoxville, Tennessee. Prior to the PET scan, patients fasted for at least six hours. Static imaging was performed 20 to 40 minutes after intravenous injection of $11 \mathrm{C}$ methionine was. For the $\mathrm{mCT}$ camera, a low dose CT was acquired for attenuation correction and images were reconstructed using Truex + TOF with 3 iterations and 21 subsets in a $400 \times 400$ matrix size (zoom 1.0). For the HR + camera, a transmission scan was performed immediately after emission scanning in the same bed positions in order to correct for attenuation and images were reconstructed using OSEM with 3 iterations and 21 subsets. The mean dosage was $205 \mathrm{MBq}$ (range 192 to $224 \mathrm{MBq}$ ), radiochemical purity was always higher than $95 \%$ and the specific activity higher than $10000 \mathrm{GBq} / \mathrm{mmol}$. The 2006 EANM procedure guidelines for brain tumor imaging using labeled amino acid analogues were followed [18].

\section{Image analysis}

The different volumes of interest ( $\mathrm{VOI}$ ), including contrast enhancing tumor and peritumoral edema were delineated manually. This delineation was performed on the available preoperative MRI using 3DSlicer version 4.10.2 (http://www.slicer.org). The primary tumor was delineated as the contrast enhancing area on T1, internal cystic or necrotic tissue was not excluded from the primary tumor VOI. Peritumoral area was determined using the T2 FLAIR sequence, it was defined as the area of high intensity surrounding the tumor, excluding the primary tumor $\mathrm{VOI}$ as already determined on the post-contrast T1 weighted sequence. These delineations were made manually on a slice by slice basis as illustrated on Fig. 1A\&B. Besides these sequences, unenhanced T1 and T2 were also used to support assessment. The determined VOls were then used as a supporting tool in PET analysis using PMOD version 4.1 (PMOD Technologies, Zurich, Switzerland). PET scans were co-registered to corresponding MRI, making it possible to use the MRI for overlays on the PET. Using the semiautomatic delineation tools in PMOD, the primary tumor as visible on T1 post-contrast weighted MRI was converted into a mask, which could be overlaid on the PET scan (Fig. 1C\&D). These VOls were compared to the predetermined delineations of 3DSlicer and adjusted where necessary. Additionally, the high tracer uptake PET lesion was also delineated semi automatically using PMOD software. The mask was used to divide the high PET uptake lesion into two parts: 1) the high PET uptake lesion inside the mask and 2) the high PET uptake lesion outside the mask.

\section{PET parameters}

PET parameters were collected from these VOls separately, where parameters from inside the mask were defined as those of the primary tumor and parameters from outside the mask were defined as those of the peritumoral area. Several PET parameters were determined within these two VOls and included maximum and mean tumor-to-normal-ratios (TNRmax and TNRmean, respectively) as metabolic parameters and metabolic tumor volume (MTV) as volumetric parameter. For each voxel, the standardized uptake value (SUV) was determined as follows.

SUV $=\mathrm{C}(\mathrm{T}) /[$ injection dose $(\mathrm{MBq}) /$ patient weight $(\mathrm{kg})][19]$

The TNR values were calculated by dividing the corresponding SUV value within the VOI by the SUV value within a $1-\mathrm{cm}$ radius sphere mirrored on the contralateral centrum semiovale. MTV represents the volume 
of the PET enhancing area in $\mathrm{mL}$.

\section{Statistics}

All statistical analysis was performed in SPSS version 23.0 (Armonk, NY, IBM Corp.). Patient characteristics were compared using the corresponding non-parametric statistical tests. Proportions were compared using a chi square test and medians were compared with a Mann Whitney $U$ test. Median values of the PET parameters and tumor volumes were determined and differences in rankings were tested using the Mann Whitney $U$ test. Statically significance was set at a two-sided $p$-value for the general patient characteristics. As we hypothesized more aggressive features in ventricle contacting tumors, we used a one-sided $p$ value of 0.05 for these analyses.

\section{Results}

\section{Patient Population}

The 12 included patients had a median age of 59 years (range 41-73). No significant differences were observed between the two groups. The characteristics and associated significance values are shown in Table 1.

Table 1

- Comparing patient characteristics of the ventricle contacting group with the non-contacting group

\begin{tabular}{|c|c|c|c|c|}
\hline & & \multicolumn{3}{|c|}{ VENTRICLE CONTACT } \\
\hline & & No & Yes & Sig. \\
\hline \multicolumn{2}{|l|}{ Median age at diagnosis in years (range) } & $\begin{array}{l}61(47- \\
73)\end{array}$ & $\begin{array}{l}52(41- \\
63)\end{array}$ & 0.222 \\
\hline \multirow[t]{2}{*}{$\operatorname{Sex}(N)$} & Female & 2 & 2 & 0.679 \\
\hline & Male & 5 & 3 & \\
\hline \multirow[t]{3}{*}{ Resection $(\mathrm{N})$} & $\begin{array}{l}\text { Gross } \\
\text { total }\end{array}$ & 3 & 2 & 0.152 \\
\hline & Subtotal & 0 & 2 & \\
\hline & Biopsy & 4 & 1 & \\
\hline \multirow[t]{2}{*}{ Use of corticosteroids at time of metPET $(\mathrm{N})$} & Yes & 1 & 3 & 0.098 \\
\hline & No & 6 & 2 & \\
\hline \multicolumn{2}{|l|}{$\begin{array}{l}\text { Median time between PET and MRI in days } \\
\text { (range)_ }\end{array}$} & $9(4-23)$ & $10(0-29)$ & 1.000 \\
\hline
\end{tabular}


After scoring these patients for ventricle contact, there was only one disagreement resulting in a good inter-rater agreement with a kappa of 0.80 (20). Of the 12 included patients, 5 were found to have a ventricle contacting tumor (38.5\%), while 7 had a non-contacting tumor (61.5\%).

\section{$11 \mathrm{C}$ methionine PET Parameters}

All PET parameters of both the primary tumor VOI and the peritumoral VOI demonstrated higher median values for the ventricle contacting group compared to the non-contacting group, as well as higher mean ranks (Tables 2 and 3). Higher metabolism was shown within the primary tumor VOI with a statistically significant higher TNRmean of 4.44 (range 2.94-7.74) for the ventricle contacting group versus 2.67 (range 1.32-3.44) for the non-contacting group, $p=0.015$. Although significance values came very close for TNRmax, the differences between the tumor groups were non-significant $(p=0.053)$. MTV was not significantly different between the ventricle contacting and non-contacting group. Despite that all peritumoral VOI parameters were higher in the ventricle contacting group, none of the differences showed statistically significance, with p-values ranging from 0.074 to 0.217 .

Table 2

- PET parameters of primary tumor volume of interest

\begin{tabular}{|c|c|c|c|c|c|c|c|}
\hline & \multirow[b]{2}{*}{$\begin{array}{l}\text { Ventricle } \\
\text { contact }\end{array}$} & \multirow[b]{2}{*}{$\mathrm{n}$} & \multirow[b]{2}{*}{$\begin{array}{l}\text { Median score } \\
\text { (range) }\end{array}$} & \multicolumn{4}{|c|}{ Mann Whitney U test results } \\
\hline & & & & $\begin{array}{l}\text { Mean } \\
\text { rank }\end{array}$ & $\begin{array}{l}\text { Sum of } \\
\text { ranks }\end{array}$ & $\begin{array}{l}Z \\
\text { value }\end{array}$ & Sig. \\
\hline \multirow[t]{2}{*}{ TNRmax } & Yes & 5 & $4.70(2.57-6.17)$ & 8.60 & 43 & -1.705 & 0.053 \\
\hline & No & 7 & $2.70(1.72-3.22)$ & 5.00 & 35 & & \\
\hline \multirow[t]{2}{*}{ TNRmean } & Yes & 5 & $4.44(2.94-7.74)$ & 9.20 & 46 & -2.192 & 0.015 \\
\hline & No & 7 & $2.67(1.32-3.44)$ & 4.57 & 32 & & \\
\hline \multirow[t]{2}{*}{$\begin{array}{l}\text { MTV } \\
\text { (cm3) }\end{array}$} & Yes & 5 & $\begin{array}{l}6.75(0.22- \\
13.83)\end{array}$ & 8.60 & 43 & -1.705 & 0.053 \\
\hline & No & 7 & $0.39(0.05-3.38)$ & 5.00 & 35 & & \\
\hline
\end{tabular}


Table 3

- PET parameters of peritumoral area VOI

\section{Mann Whitney U test results}

\begin{tabular}{|c|c|c|c|c|c|c|c|}
\hline & $\begin{array}{l}\text { Ventricle } \\
\text { contact }\end{array}$ & $\mathrm{n}$ & $\begin{array}{l}\text { Median score } \\
\text { (range) }\end{array}$ & $\begin{array}{l}\text { Mean } \\
\text { rank }\end{array}$ & $\begin{array}{l}\text { Sum of } \\
\text { ranks }\end{array}$ & $\begin{array}{l}Z \\
\text { value }\end{array}$ & Sig. \\
\hline \multirow[t]{2}{*}{ TNRmax } & Yes & 5 & $5.08(1.96-6.13)$ & 8.20 & 41 & -1.380 & 0.101 \\
\hline & No & 7 & $2.70(1.94-3.29)$ & 5.29 & 37 & & \\
\hline \multirow[t]{2}{*}{ TNRmean } & Yes & 5 & $4.62(2.48-5.38)$ & 8.40 & 42 & -1.543 & 0.074 \\
\hline & No & 7 & $2.91(1.35-3.60)$ & 5.14 & 36 & & \\
\hline \multirow[t]{2}{*}{$\begin{array}{l}\text { MTV } \\
\text { (cm3) }\end{array}$} & Yes & 5 & $\begin{array}{l}10.84(4.39- \\
28.18\end{array}$ & 8.20 & 41 & -1.380 & 0.101 \\
\hline & No & 7 & $\begin{array}{l}3.06(0.68- \\
36.14)\end{array}$ & 5.29 & 37 & & \\
\hline
\end{tabular}

\section{Discussion}

This is the first study comparing metPET characteristics of GBM in relation to ventricle contact. Our results show an increased methionine uptake for ventricle contacting GBMs in comparison to noncontacting GBMs. Specifically, our results demonstrated significantly higher TNRmean for ventricle contacting tumors than non-contacting tumors. This would imply an increased protein synthesis and therefore higher proliferation in the ventricle contacting group, further supporting the more aggressive characteristics of ventricle contacting GBMs.

Ventricle contact in GBM has previously been associated with several imaging characteristics related to tumor aggressiveness, such as larger tumor volumes, more multifocal and distant recurrences, and higher peritumoral perfusion, all of which potentially contribute to the lower survival of these tumors $[6,9,10]$. In addition to this, we have now also demonstrated a higher methionine uptake, as a measure of increased proliferation, for ventricle contacting GBM.

Although our study is the first to study metPET in relation to ventricle contact in GBM, our results are in line with a recent study looking at cellular proliferation with 0-(2-[18F]fluoroethyl)-L-tyrosine (FET) PET [21]. Although it was not the primary research question, FET uptake showed a significantly higher SUVmean value for GBM with ventricle involvement when compared to GBM without ventricle contact.

We also hypothesized that ventricle contact in GBM could be related to higher methionine uptake levels in the peritumoral area. The peritumoral area plays an important role in the recurrence of GBM as it contains 
both vasogenic edema and infiltrating tumor cells. The peritumoral area is of particular interest in relation to ventricle contact in GBM as one characteristic of ventricle contacting GBM is a more distant and multifocal recurrence $[6,10]$.

In addition, a recent study showed a higher peritumoral perfusion for ventricle contacting GBMs, associated with a more aggressive peritumoral infiltration [22]. In line with these hypotheses, we did find higher values for metPET parameters in the peritumoral region, but none of those differences reached significance.

Despite the significant difference in TNRmean between ventricle contacting and non-contacting GBMs, this study does have its limitations. The first limitation is the small sample size. This fact is a direct cause for the inability to meet assumptions for normal distribution and asymptotic significance, as well as a limited power of the outcomes. It is because of this that this study should primarily be seen as a pilot study and an invitation to perform larger prospective studies. Nevertheless, a significant difference for TNRmean has been observed, even with this limited sample size. Furthermore, the other PET parameters point in the same direction although not reaching significance. A second limitation is the retrospective nature of our data, resulting in the lack of a standardized protocol, such as the use of two PET systems with different resolutions. These different resolutions could potentially have introduced partial volume effects. However, by employing TNR and thus dividing uptake values by the background uptake, we aimed to limit these partial volume effects. The lack of standardization also resulted in a heterogeneous time interval between PET and MRI acquisition, which in some cases amounted to as much as 29 days. Prospective studies should make sure that time between MRI and PET is minimalized, which would lead to a smaller inaccuracy when performing the MRI overlay on the PET.

\section{Conclusion}

Ventricle contacting GBMs demonstrate a significantly higher uptake of methionine than non-contacting tumors. The increased uptake of methionine indirectly indicates a higher proliferation. These findings possibly explain the survival difference between ventricle contacting and non-contacting GBMs and should be considered in treatment decisions.

\section{Declarations}

Funding: No funding was received for conducting this study.

Conflicts of interest: The authors have no conflicts of interest to declare that are relevant to the content of this article.

Availability of data and material: The data generated and analyzed during the current study are available from the corresponding author on reasonable request. 
Authors' contributions: All authors contributed to the study conception and design. Material preparation, data collection and analysis were performed by Bart R.J. van Dijken, Bram Schuuring, Gilles N.

Stormezand and Anouk van der Hoorn. The first draft of the manuscript was written by Bram Schuuring and Bart R.J. van Dijken and all authors commented on previous versions of the manuscript. All authors read and approved the final manuscript.

Ethics approval: This study was approved by the local Ethics Committee of the University Medical Center Groningen and the need for obtaining informed consent was waived in view of the retrospective nature of the study.

\section{References}

1. Witthayanuwat, S, Pesee, M, Supaadirek, C, Supakalin, N, Thamronganantasakul, K, Krusun, S. (2018) Survival Analysis of Glioblastoma Multiforme. Asian Pacific Journal of Cancer Prevention. 19(9): 2613-2617.

2. Stupp R, Mason WP, van den Bent MJ, et al (2005) Radiotherapy plus concomitant and adjuvant temozolomide for glioblastoma. N Engl J Med 352:987-996.

3. Barajas RF Jr, Phillips JJ, Parvataneni R, et al. (2012) Regional variation in histopathologic features of tumor specimens from treatment-naïve glioblastoma correlates with anatomic and physiologic MR Imaging. Neuro Oncol. 14(7):942-54.

4. Rathore S, Akbari H, Doshi J, et al. (2018) Radiomic signature of infiltration in peritumoral edema predicts subsequent recurrence in glioblastoma: implications for personalized radiotherapy planning. J Med Imaging (Bellingham). 5(2):021219.

5. Akbari H, Macyszyn L, Da X, et al. (2014) Pattern analysis of dynamic susceptibility contrastenhanced MR imaging demonstrates peritumoral tissue heterogeneity. Radiology. 273(2):502-10.

6. Mistry AM, Hale AT, Chambless LB, Weaver KD, Thompson RC, Ihrie RA. (2017) Influence of glioblastoma contact with the lateral ventricle on survival: a meta-analysis. J Neurooncol. 131(1):125-133.

7. Galli R, Binda E, Orfanelli U, et al. (2004) Isolation and characterization of tumorigenic, stem-like neural precursors from human glioblastoma. Cancer Res. 64(19):7011-7021.

8. Sanai N, Alvarez-Buylla A, Berger MS. (2005) Neural stem cells and the origin of gliomas. N Engl J Med. 353:811-822.

9. Berendsen S, van Bodegraven E, Seute T, et al. (2019) Adverse prognosis of glioblastoma contacting the subventricular zone: Biological correlates. PLoS One. 14(10):e0222717.

10. Lim DA, Cha S, Mayo MC, et al. (2007) Relationship of glioblastoma multiforme to stem cell regions predicts invasive and multifocal tumor phenotype. Neuro Oncol. 9:424-429.

11. Glaudemans, AWJM, Enting, RH, Heesters, MAAM et al. (2013) Value of 11C-methionine PET in imaging brain tumours and metastases. Eur J Nucl Med Mol Imaging 40:615-635. 
12. Bergstrom M, Lundqvist $H$, Ericson $K$, et al. (1987) Comparison of the accumulation kinetics of L(methyl-11C)-methionine and D-(methyl-11C)-methionine in brain tumors studied with positron emission tomography. Acta Radiol. 28:225-9.

13. Kubota K. (2001) From tumor biology to clinical PET: a review of positron emission tomography (PET) in oncology. Ann Nucl Med. 15:471-86.

14. Weller, M, van den Bent, M, Preusser, M. et al.(2020) EANO guidelines on the diagnosis and treatment of diffuse gliomas of adulthood. Nat Rev Clin Oncol. Epub ahead of print.

15. Cohen, J. (1960) A coefficient of agreement for nominal scales. Educational and psychological Measurement. 20.1: 37-46.

16. Liang TH, Kuo SH, Wang CW, et al. (2016) Adverse prognosis and distinct progression patterns after concurrent chemoradiotherapy for glioblastoma with synchronous subventricular zone and corpus callosum invasion. Radiother Oncol. 118:16-23.

17. Chen L, Chaichana KL, Kleinberg L, Ye X, Quinones-Hinojosa A, Redmond K. (2015) Glioblastoma recurrence patterns near neural stem cell regions. Radiother Oncol. 116:294-300.

18. Vander Borght T, Asenbaum S, Bartenstein P, et al. (2006) EANM procedure guidelines for brain tumour imaging using labelled amino acid analogues. Eur J Nucl Med Mol Imaging. 33:1374-1380.

19. Kim EE, Lee MC, Innoue T, Wang WH. (2012) Clinical PET and PET/CT. 2nd ed. New York: Springer; EAN: 9781441908025 p42.

20. Landis JR, Koch GG. (1977) The measurement of observer agreement for categorical data. Biometrics. 33(1):159-174.

21. Harat M, Małkowski B, Roszkowski K. (2019) Prognostic value of subventricular zone involvement in relation to tumor volumes defined by fused MRI and O-(2-[18F]fluoroethyl)-L-tyrosine (FET) PET imaging in glioblastoma multiforme. Radiat Oncol. 14(1):37.

22. van Dijken BRJ, van Laar PJ, Li C et al. Ventricle contact is associated with lower survival and increased peritumoral perfusion in glioblastoma. J Neurosurg. 131(3):717-723.

\section{Figures}



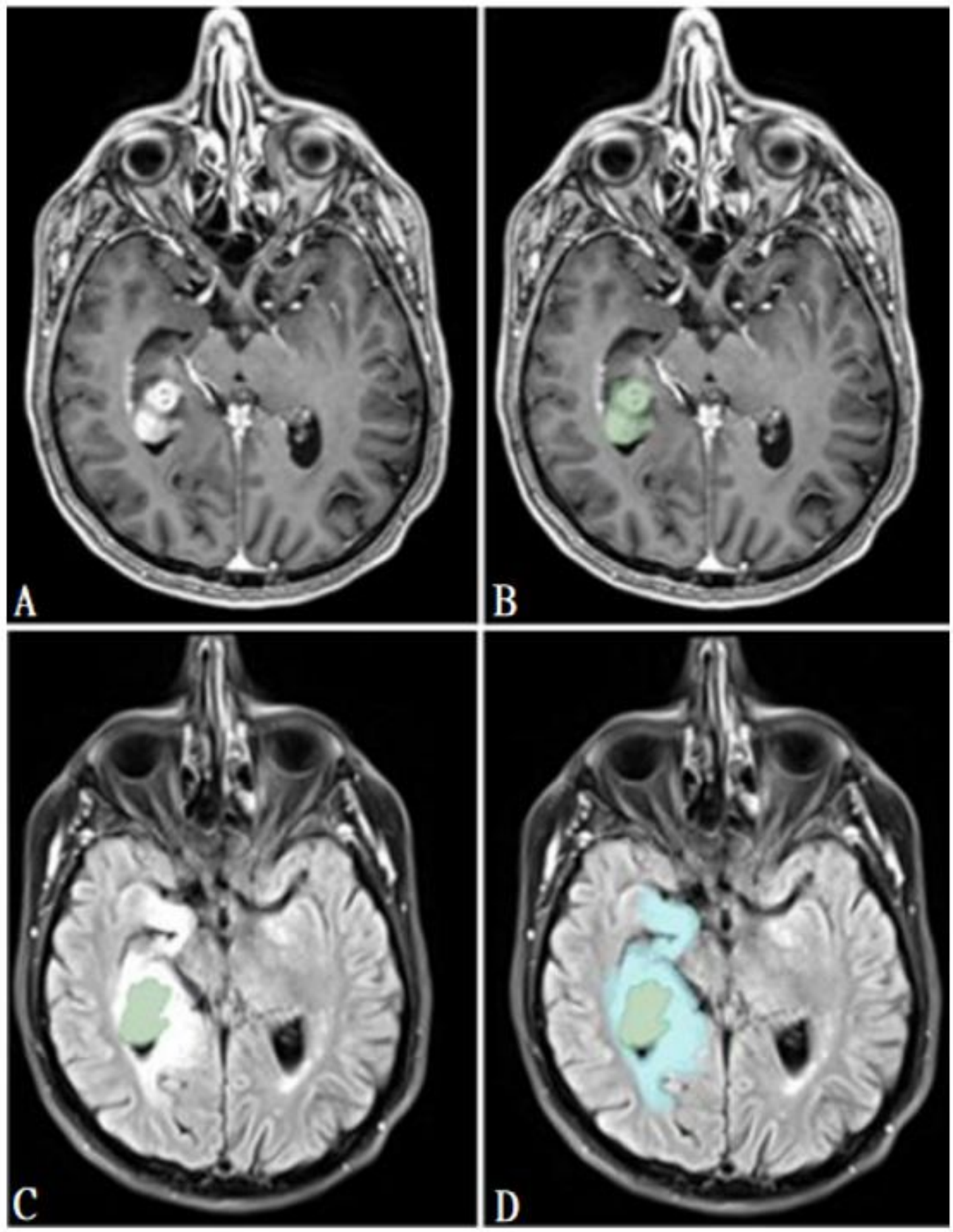

\section{Figure 1}

Segmentation of MRI lesions A (upper left): Post-contrast T1 weighted MRI before segmentation demonstrating a right mesiotemporal ventricle contacting GBM; B (upper right): The same slice after segmentation of the contrast enhancing lesion (green mask); C (lower left): FLAIR sequence with the primary tumor segmentation overlay (green mask), but before peritumoral edema is segmented; D (lower right): The same slice after peritumoral edema segmentation. 

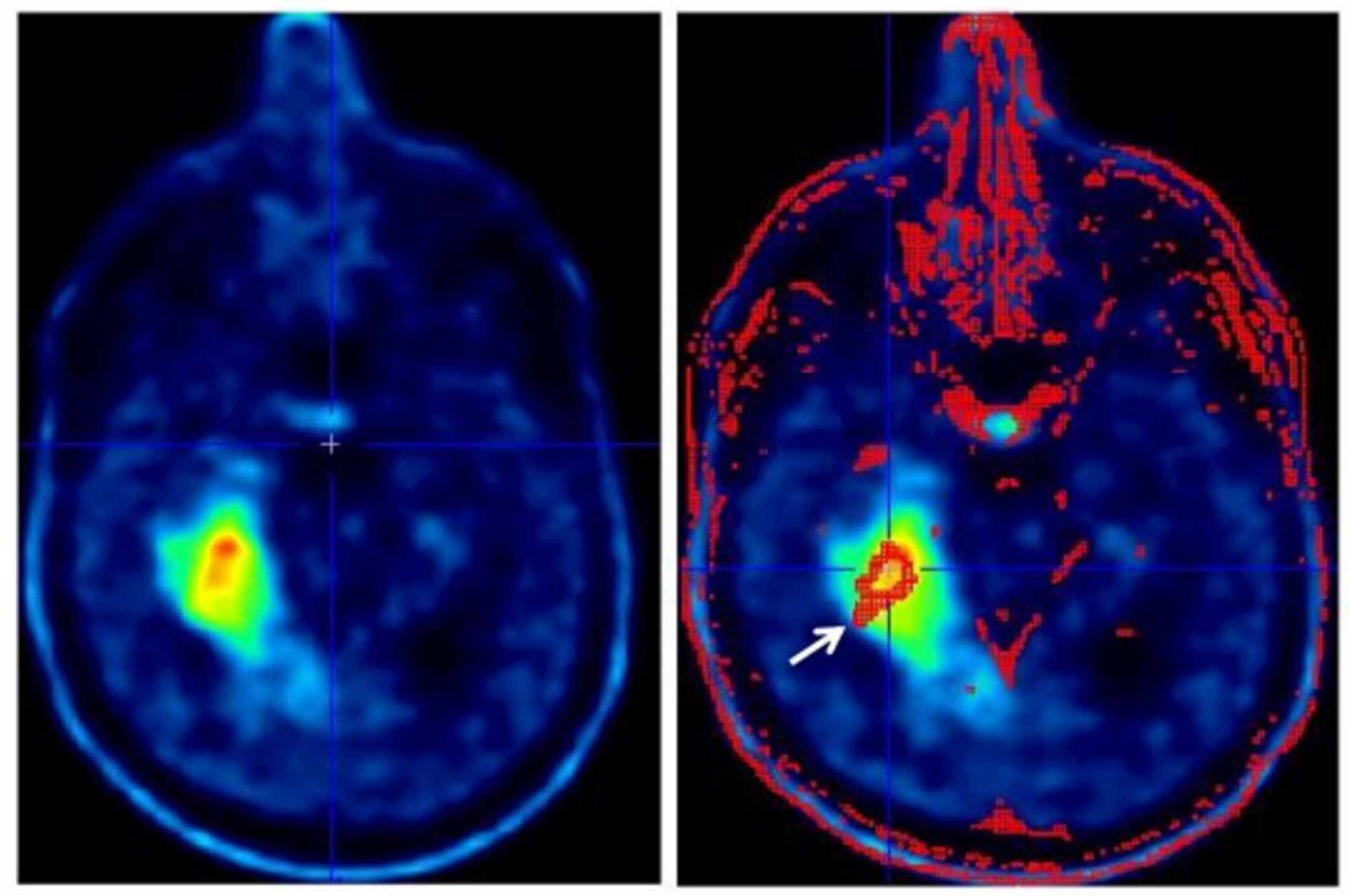

\section{Figure 2}

Segmentation of metPET volumes of interest (VOI) MetPET images of the same patient as figure 1, before (left) and after masking with MRI (right). The mask based on the contrast enhancing lesion is indicated with an arrow. The high uptake area inside this mask was used as the primary tumor VOI and the high uptake area outside of it was used as the peritumoral area VOI. If necessary, masking was adjusted to match manual segmentations.

\section{Supplementary Files}

This is a list of supplementary files associated with this preprint. Click to download.

- APPENDIXTABLESANDFIGURES.docx 